\title{
Effects of Operational Conditions on Electrochemical Water Softening Using DSA Anode
}

\author{
Jiaoping Wang ${ }^{1,}$, Meina Shi', b and Gang Zhang ${ }^{1, c^{*}}$ \\ ${ }^{1}$ School of Civil Engineering and Architecture, University of Jinan, Jinan 250022, China \\ ${ }^{2}$ Weihai Urban Planning \& Design Institute Co., Ltd, Weihai 264299, China \\ a920912654@qq.com, bshimina92@163.com, ’zg0107@126.com
}

\begin{abstract}
Keywords: Dimensionally stable anode; Water softening; Current density; inter-electrode distance; Electrolysis

Abstract. Hardness forming ions such as $\mathrm{Ca} 2+$ and $\mathrm{Mg} 2+$ in cooling water tended towards scale on the surface of the heat exchanger, resulting in reduction of heat transfer efficiency. In this paper, by using DSA electrodes, effects of operational conditions on water softening was evaluated by Lab scale experiments. The experimental results shown that, when the current density increased from $50 \mathrm{~A} / \mathrm{m} 2$ to $250 \mathrm{~A} / \mathrm{m} 2$, the removal rate of $\mathrm{Ca} 2+$ was improved from $14.0 \%$ to $46.3 \%$ with approximately logarithmic relationship. On the other hand, the removal rate of $\mathrm{Ca} 2+$ decreased from $71.63 \%$ to $14.91 \%$ among with the inter-electrode distance extended from $2 \mathrm{~cm}$ to $10 \mathrm{~cm}$. After uninterrupted electrolysis of raw water for 8 minutes, the removal rate increased to $58.6 \%$, and after the 8th minute, the uninterrupted electronic reaction couldn't cause further removal of hardness forming ions. XRD and SEM shown that the crystal form of calcium carbonate on the cathode plate was mainly calcite. The research results provide important practical data for the research and design of this kind of water softening system.
\end{abstract}

\section{Introduction}

Changes in the physicochemical conditions of water cause $\mathrm{Ca}^{2+}$ in the water to precipitate as calcium carbonate and adhere to the surface of the container to form scale. In industrial production, $\mathrm{Ca}^{2+}$ in water causes scaling on the surface of heat exchanger of circulating cooling water system, which will result in the decrease of heat transfer efficiency, the increase of energy consumption and the shortening of equipment life ${ }^{[1-8]}$. Electrochemical softening of circulating cooling water is to reduce the hardness of water by crystallization and precipitation the scaling ions on the cathode plate, which can greatly reduce the water recharge of the system. Dimensionally stable anode (DSA) has high electrocatalytic activity, excellent corrosion resistance and mechanical stability. The development of DSA anode materials has improved the treatment efficiency of electrochemical water treatment technology ${ }^{[9,10]}$.

The main influencing factors of electrochemical softening are current density, electrode spacing and electrolysis time. This paper will discuss the effect of the above factors on the electrochemical softening by DSA anode of circulating cooling water and explain the experimental phenomena.

$$
\begin{aligned}
& \mathrm{O}_{2}+2 \mathrm{H}_{2} \mathrm{O}+4 \mathrm{e}^{-} \rightarrow 4 \mathrm{OH}^{-} \\
& 2 \mathrm{H}_{2} \mathrm{O}+2 \mathrm{e}^{-} \rightarrow \mathrm{H}_{2}+2 \mathrm{OH}^{-} \\
& \mathrm{HCO}_{3}^{-}+\mathrm{OH}^{-} \rightarrow \mathrm{H}_{2} \mathrm{O}+\mathrm{CO}_{3}{ }^{2-} \\
& \mathrm{Ca}^{2+}+\mathrm{CO}_{3}{ }^{2-} \rightarrow \mathrm{CaCO}_{3}
\end{aligned}
$$

\section{Experiment}

Experimental system. As shown in Fig. 1, the experimental system consisted of a DC power supply and a pilot electrolyser. An electrode plate placement slot was provided every $1 \mathrm{~cm}$ in the length direction of the electrolyser to facilitate changing the inter-electrode distance. DC power supply (Yizhan Electronic Instrument Co., Ltd, Shenzhen of China) was used to supply power at a constant current intensity. The cathode was a $10 \mathrm{~cm} \times 10 \mathrm{~cm}$ titanium plated steel and the anode plate was a $10 \mathrm{~cm} \times 10 \mathrm{~cm} \mathrm{Ti} / \mathrm{IrO}_{2}-\mathrm{RuO}_{2}$ electrode. 
Raw water. $\mathrm{CaCl}_{2}$ and $\mathrm{NaHCO}_{3}$ were added to water to simulate the temporary hardness in water. The molar ratio of $\mathrm{CaCl}_{2}$ to $\mathrm{NaHCO}_{3}$ was $1: 2$. In addition, $2 \mathrm{~g}$ of $\mathrm{NaCl}$ was added per liter of water in order to elevate the conductivity. The reagents used in the experimental were produced by Hengxing Chemical Reagent Manufacturing Co., Ltd (Tianjin of China) and their grades were analytically pure.

Analysis methods. Determination of $\mathrm{Ca}^{2+}$ in water samples by EDTA complexometric titration. Three parallel samples were taken for each working condition during the experimental. The minimum detection concentration of this method is 0.05 $\mathrm{mmol} / \mathrm{L}$, and the repeatability deviation is \pm 0.04 $\mathrm{mmol} / \mathrm{L}$.

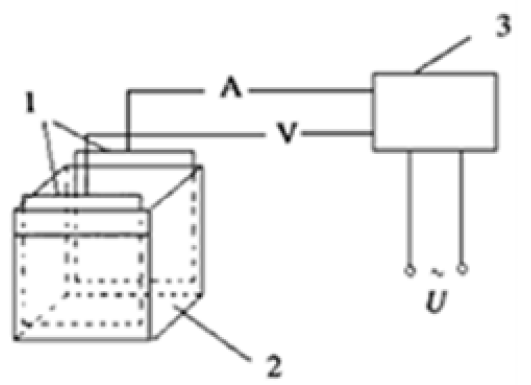

Fig.1. Electrolysis experimental system 1-Plate; 2- Pilot electrolyser ; 3-DC power supply

\section{Results and discussion}

Current density. Fig. 2 shows the change of the removal rate with respect to current density. The inter-electrode distance in the experimental was $6 \mathrm{~cm}$. When the current density increased from 50 $\mathrm{A} / \mathrm{m}^{2}$ to $250 \mathrm{~A} / \mathrm{m}^{2}$, the $\mathrm{Ca}^{2+}$ removal rate elevated from $14 \%$ to $46.3 \%$ (The electrolysis time was $5 \mathrm{~min}$.) and $26.8 \%$ to $53.5 \%$ (The electrolysis time was $10 \mathrm{~min}$.). With the increase of current density, the removal rate of $\mathrm{Ca}^{2+}$ also enhanced, but the enhancing trend decreased slowly. This phenomenon is mainly affected by mass transfer.

With increasing current density, the $\mathrm{OH}^{-}$quantities ((Eq. (1) and Eq. (2)) produced at the cathode increases according to Faradays law. Combined with reactions (3) and (4), it could be concluded that the $\mathrm{Ca}^{2+}$ removal rate increased with current density. The $\mathrm{Ca}^{2+}$ removal rate was mainly limited by the electromigration and diffusion kinetics of $\mathrm{Ca}^{2+}$ and $\mathrm{HCO}_{3}{ }^{-}$. As the current density increased, the electromigration of $\mathrm{HCO}^{-}$enhanced, which made it difficult increasingly for $\mathrm{HCO}_{3}{ }^{-}$to be enriched near the cathode by diffusion. Excess $\mathrm{OH}^{-}$did not accelerate the formation of scale on the cathode plate. This was an important reason for the slowing down of the $\mathrm{Ca}^{2+}$ removal rate enhancing trend. At high current density and $\mathrm{OH}^{-}$concentration, reaction (3) was the rate-limiting step of electrochemical removal of $\mathrm{Ca}^{2+}$ in circulating water. Secondly, the increase in current density also led to hydrogen evolution enhancing at the cathode (Eq. (2)). The cathode generated a large number of bubbles, and the disturbance to the water was very severe. This would affect the mass transfer of $\mathrm{HCO}^{-}$to the cathode plate under the condition of longer inter-electrode distance $(6 \mathrm{~cm})$.

Inter-electrode distance. The distance between the cathode and anode is also a major parameter. Fig. 3 shows the variation of calcium removal rate with the increase of inter-electrode distance. It could be observed that the variation of the inter-electrode distance has a significant effect on the $\mathrm{Ca}^{2+}$ removal rate. When the inter-electrode distance was $2 \mathrm{~cm}$, the hardness removal rate was $71.63 \%$; when the inter-electrode distance was $10 \mathrm{~cm}$, the hardness removal rate was only $14.91 \%$. There were two reasons why the $\mathrm{Ca}^{2+}$ removal rate decreased with increasing inter-electrode distance: $\Phi$ When the inter-electrode distance decreased, $\mathrm{Ca}^{2+}$ and $\mathrm{HCO}_{3}{ }^{-}$present in the bulk phase could diffuse to the cathode reaction zone more quickly. Chemical reactions (3) and (4) could take place here, so that the $\mathrm{Ca}^{2+}$ in the bulk phase was exhausted more completely. $\theta$ The hydrogen on the cathode, the chlorine and oxygen on the anode had disturbances to the solution. Under the condition that the distance between the plates was close, this disturbance could cover the entire bulk phase and enhanced the mass transfer of ions from the solution to the two poles. In the case of large plate spacing, the gas disturbance only affected the area near the plate and did not disturb the area far from the plate, which has an adverse effect on the mass transfer of $\mathrm{HCO}_{3}{ }^{-}$to the cathode in the more distant areas. 
Electrolysis time. The electrolysis time is another important factor of the process. Fig. 5 shows how $\mathrm{Ca}^{2+}$ removal rate changes with the passage of electrolysis time. It could be seen that the $\mathrm{Ca}^{2+}$ removal rate enhanced with the increase of the electrolysis time, and as the electrolysis time advances, the $\mathrm{Ca}^{2+}$ removal rate enhanced more and more slowly, reached $58.6 \%$ at 8 minutes. Then as the electrolysis time increased, the removal rate of $\mathrm{Ca}^{2+}$ was almost never changed.

From Faradays law, it can be seen that as the electrolysis time increases, the concentration of $\mathrm{OH}^{-}$ in the solution continuously enhances, so that the removal rate of $\mathrm{Ca}^{2+}$ continued to elevate. With the constant consumption of $\mathrm{Ca}^{2+}$ and $\mathrm{HCO}_{3}{ }^{-}$in the solution, the chemical reaction rates of (3) and (4) decreased. Combined with mass transfer in the bulk phase and hydrogen evolution of the cathode, there is less and less $\mathrm{HCO}_{3}{ }^{-}$enriched into the cathode plate. The increasing trend of $\mathrm{Ca}^{2+}$ removal rate was more and more gentle with time. When the reaction was carried out for 8 minutes, almost no $\mathrm{HCO}_{3}{ }^{-}$reacted with $\mathrm{OH}^{-}$in the cathode reaction zone. Therefore, as the electrolysis time and $\mathrm{OH}^{-}$concentration increased, the $\mathrm{Ca}^{2+}$ removal rate did not change.

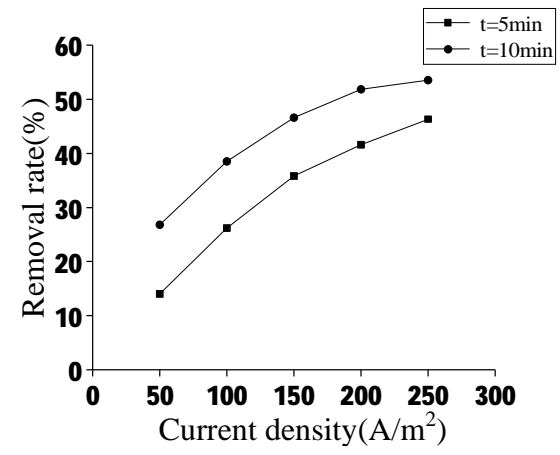

Fig.2. Effect of current intensity on removal rate

Scale of the cathode. After experimental test, the morphology of the scale was very regular (Fig. 5b). According to $\mathrm{X}$ ray diffraction the scale was mainly calcite (Fig. 5a). Calcite scale texture is hard and extremely difficult to clean up, which causes great problems for the practical engineering application of equipment. The research of cathode cleaning method has become the focus of many scholars ${ }^{[3,12]}$. There-fore, seeking an ideal cathode plate scale cleaning method is the next research direction.

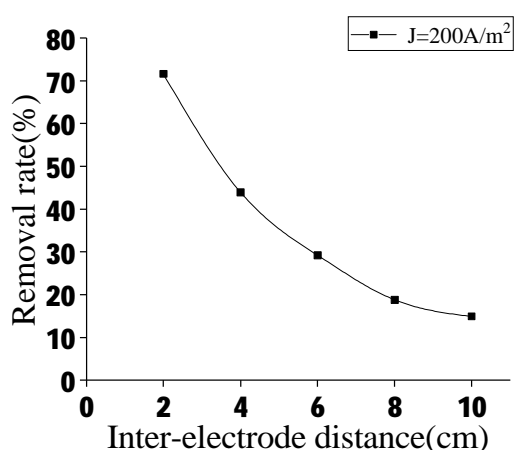

Fig.3. Effect of inter-electrode distance on removal rate

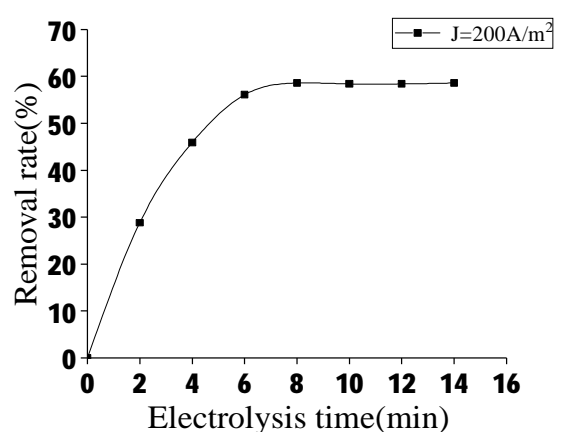

Fig.4. Effect of electrolysis time on removal rate

\section{Results and discussion}

The present study indicated that calcite precipitation occured through a chemical process following the electrochemical generation of $\mathrm{OH}^{-}$ions in the vicinity of the electrode surface. With the continuous increase of current density, the removal rate of $\mathrm{Ca}^{2+}$ also enhanced, reaching a maximum of $53.5 \%$. Due to the effect of mass transfer and acidification of scale-forming ions in the pilot electrolyser, the increasing trend of the $\mathrm{Ca}^{2+}$ removal rate tended to be gradual. The smaller the distance between the plates, the faster the diffusion of $\mathrm{Ca}^{2+}$ and $\mathrm{HCO}^{3-}$ to the cathode reaction zone, which made the $\mathrm{Ca}^{2+}$ removal rate decrease with the increase of the plate spacing. In the experiment, the distance between plates increased from $2 \mathrm{~cm}$ to $10 \mathrm{~cm}$, and the removal rate of $\mathrm{Ca} 2+$ decreased from $71.63 \%$ to $14.91 \%$. For a smaller inter-electrode distance, the bubbles produced by the reaction between the two poles are conducive to the diffusion of scale ions. For longer inter-electrode distance, the bubbles produced by two-pole reaction have an adverse effect on the enrichment of scale ions to the cathode. With the prolongation of electrolysis time, $\mathrm{Ca}^{2+}$ and $\mathrm{HCO}^{3-}$ 
in the solution were continuously depleted, and the rate of chemical reaction producing $\mathrm{CaCO} 3$ was continuously reduced, so that the trend of increasing $\mathrm{Ca}^{2+}$ removal rate was flattened, and basically did not change after 8 minutes reaching $58.6 \%$.

The experimental tests performed on a pilot electrolyser can understand the behavior of an industrial device of circulating cooling water softening more deeply. The information on the specific effects of operational condition such as current density, inter-electrode distance and electrolysis time will be very precious to improve the softening efficiency of an industrial equipment.

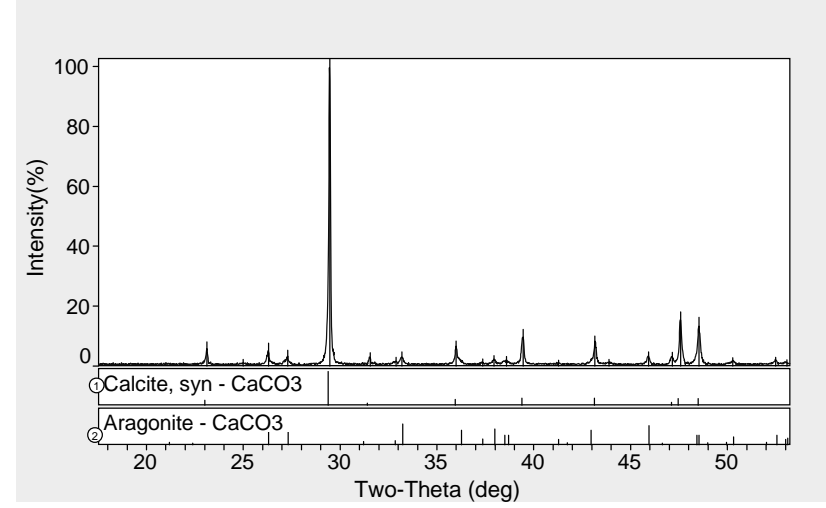

(a) $\mathrm{X}$ ray diffraction diagram

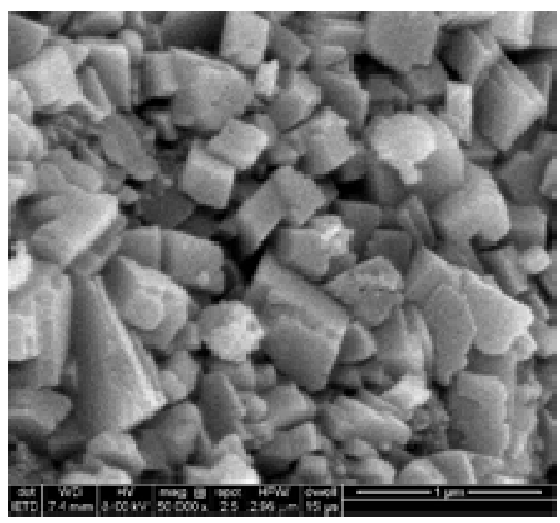

(b) SEM image

Fig.5. Scale precipitated on the cathode of the pilot electrolyser

\section{References}

[1] C. Shen, C. Cirone, A.M. Jacobi, et al: Appl. Therm. Eng Vol. 79 (2015), p.74-87.

[2] D.E. Abd-El-Khalek, B.A. Abd-El-Nabey: Desalination Vol. 311(5) (2013), p.227-233.

[3] D. Hasson, G. Sidorenko, R. Semiat: Desalination Vol. 263(1) (2010), p.285-289.

[4] S.H. Lee, Y.I. Cho: Int. J. Heat and Mass Transfer Vol. 45 (2010), p.4163-4174.

[5] P. Shakkthivel, T. Vasudevan: Desalination Vol. 197 (2006), p.179-189.

[6] Kh. Rahmani, R. Jadidian, S. Haghtalab: Desalination Vol. 393 (2016), p.174-185.

[7] S. Seo, H. Jeon, J.K. Lee, et al: Water Res Vol. 44(7) (2015), p.2267-2275.

[8] S. Zhi, S. Zhang,: Desalination Vol. 349 (2014), p.68-72.

[9] S. Dbira, N. Bensalah, P. Cañizares, et al: J. Electroanal. Chem Vol. 744 (2015), p.62-68.

[10]E. Turro, A. Giannis, R. Cossu, et al: J. Hazard. Mater Vol. 190(1) (2011), p.460-465.

[11]The State Environmental Protection Administration, The Water and Wastewater Monitoring Analysis Method Editorial Board: Water and Wastewater monitoring analysis method. 4th Edition, Beijing: China Environmental Science Press (2002), 415-418. (in Chinese)

[12] Y. Yu, H. Jin, P. meng, et al: Sep. Purif. Technol Vol. 191 (2018), p.216-224. 